\title{
The Relationship between Epidemiologic Factors and Usage Pattern of Hair Care Products in Korea
}

\author{
Kyung-Duck Park, Byung Cheol Park ${ }^{1}$, Ki Hyun Sung ${ }^{1}$, Eunyoung Lee ${ }^{2}$, Taewon Lee ${ }^{3}$, Daeki Yoon ${ }^{3}$, \\ Kyu Bong Kim ${ }^{4}$, Gyeong Hun Park ${ }^{5}$, Il Yong Park ${ }^{6}$, Myung Hwa Kim ${ }^{1}$ \\ Department of Dermatology, Kyungpook National University School of Medicine, Daegu, ${ }^{1}$ Department of Dermatology, College of \\ Medicine, Dankook University, Cheonan, ${ }^{2}$ Skin Research Institute, IEC Korea, Suwon, ${ }^{3}$ Data Computational Sciences, Division of Applied \\ Mathematical Sciences, College of Science and Technology, Korea University, Sejong, ${ }^{4}$ Department of Pharmacy, College of Pharmacy, \\ Dankook University, Cheonan, ${ }^{5}$ Department of Dermatology, Dongtan Sacred Heart Hospital, Hallym University College of Medicine, \\ Hwaseong, ${ }^{6}$ Department of Biomedical Engineering, College of Medicine, Dankook University, Cheonan, Korea
}

Background: For hair care products that are used almost everyday, it is important to estimate the cumulative dosage of long-term exposure and to assess the effects on the human body. Little data are available to evaluate actual daily usage in Asian populations. Objective: Reliable exposure data for hair care products is essential to conduct safety assessments. Methods: We evaluated the actual usage pattern and amounts by checking the daily log over a 2-week period, to obtain all the data regarding the participants' hair care preferences. And, statistical analyses were conducted to analyze the daily use amount $(\mathrm{g} / \mathrm{d})$ and daily usage per hair length $(\mathrm{g} / \mathrm{cm} / \mathrm{d})$, and other variables by sex, age group, and hair oiliness. Results: Throughout this study, we found that female users consumed significantly larger daily amounts of shampoo and rinse. Male groups used more hair gel and spray than female groups. Interestingly, all the hair care products studied scored higher levels of usage among men when calibrated per unit length. Koreans tend to use lesser amount of rinse although their hairs are usually thicker than the

Received September 28, 2018, Revised December 17, 2018, Accepted for publication January 3, 2019

Corresponding author: Myung Hwa Kim, Department of Dermatology, College of Medicine, Dankook University, 201 Manghyang-ro, Dongnamgu, Cheonan 31116, Korea. Tel: 82-41-552-3952, Fax: 82-41-552-7541, E-mail: ivymyung@dankook.ac.kr ORCID: https://orcid.org/0000-0002-9072-201X

This is an Open Access article distributed under the terms of the Creative Commons Attribution Non-Commercial License (http://creativecommons. org/licenses/by-nc/4.0) which permits unrestricted non-commercial use, distribution, and reproduction in any medium, provided the original work is properly cited.

Copyright (c) The Korean Dermatological Association and The Korean Society for Investigative Dermatology
Western hairs. Conclusion: This study provides exposure information for commonly used hair care products, which will be useful for risk assessment purposes. (Ann Dermatol 31(3) 307 314, 2019)

\section{-Keywords-}

Exposure assessment, Hair care product, Korean usage, Usage patterns

\section{INTRODUCTION}

Hair care products for personal hygiene and beauty of scalp and body hair are popular worldwide. In the past, hair cosmetics had been used for hygiene and cleanliness, but recently they have been used for various purposes such as preventing or restoring hair and scalp damage, and managing hair style. Various chemicals and natural extracts are being used for these purposes, and new challenging materials are being competitively developed today. Some ingredients have the possibility to cause harmful reactions to the body by long-term use, and in fact some preservatives are concerned about their carcinogenicity. For products that are used almost everyday, it is important to estimate the cumulative dosage of long-term exposure and to assess the effects on the human body. Therefore, we need to evaluate their harmful or toxic effects on the body by checking their usage pattern and the total exposure levels of users. To assess exposure and risk for consumers, the exposure factors of consumer products should be determined. Exposure assessments require information regarding exposure factors, such as frequency of use and 
amount of product used per event, as well as the circumstances of use ${ }^{1}$. Exposure factors have been investigated previously in a United States (US) female population of regular product users ${ }^{2,3}$, in California households ${ }^{4}$, and in Europe $^{5-11}$, and Korea ${ }^{12}$. However, the exposure data of hair care products are not uniform in previous studies and few studies have been published regarding realistic exposure factors. In addition, little data are available to evaluate actual daily usage in Asian populations.

In this study, we evaluated the actual usage pattern and amounts by checking the daily log over a 2-week period, to obtain all the data regarding the participants' hair care preferences. Therefore, the present study may facilitate development of appropriate regulatory policy and establish safety guidelines to assess the safe use of hair care products.

\section{MATERIALS AND METHODS}

\section{Study design}

This study was a prospective observational study to measure the amounts of cosmetics usage. In this study, we recruited subjects nationwidely from Dankook University Hospital, and IRB approval was obtained according to this (DKUH 2015-12-011-002). The study was conducted for two weeks each in May 2016. Subjects were recruited from Seoul (148 participants), Incheon (74 participants), Daejeon (74 participants), Daegu (74 participants), and Busan (74 participants), the five major cities in Korea. All participants were recruited using a screening questionnaire. The survey contained questions on demographics including age, sex, income, occupation, and usage pattern and types of each cosmetic product including hair care products. Among the respondents, we conducted the research on regular hair care products users. The number of participants who completed the study was variable (54 432 participants) depending on the hair care product. We excluded subjects with occupations of market research, advertising, sales promotions, public relations, cosmetics sale. In addition, anyone who recently underwent or had planned for surgery, treatment, pregnancy, move, and vacation during the study period was excluded. We evaluated the usage pattern by sex, age, hair length and scalp hair oiliness (dry, neutral, oily). The age groups included the following ranges: 10 to 19,20 to 29,30 to 39 , 40 to 49 , and over 49 . The hair length involved the longest hair starting from the top of the scalp in the present study. Hair oiliness measurements included participant questionnaires and a physician's examination.

\section{Data collection}

Seven types of hair care products were investigated in this study, including shampoo, rinse, essence, tonic, and hair style products (hair wax, gel, and spray). The participants were instructed to visit the designated hospital, bringing their hair care products. During the first visit, participants were enrolled; they also completed the consent form, and responded to the survey regarding the demographic and socioeconomic factors, including age, sex, income, occupation, facial skin and hair oiliness type (dry, neutral, oily), marital status, and body weight. Participants were also advised to use their own products as usual, not to share with anyone else, and to keep diaries to record detailed daily usage information, such as frequency, application area, and leave-on time over the two-week period. Preand post-weights of all test products were determined using a high-precision scale. Subjects weighed their actually used products under the supervision of the dispatched researchers. We confirmed the daily usage based on the participants' daily records over two weeks.

\section{Data analysis}

All statistical analyses were conducted using statistical software $\mathrm{R}$ version 3.4.3 (The R Foundation for Statistical Computing, Vienna, Austria). The 3-way and 2-way ANOVA tests were used to analyze the daily use amount $(\mathrm{g} / \mathrm{d})$ and daily usage per hair length $(\mathrm{g} / \mathrm{cm} / \mathrm{d})$, and other variables by sex, age group, and hair oiliness. Since the usage distribution did not follow the normal distribution, the ANOVA was performed after the data transformation by ordered quantile normalizing transformation or Yeo-Johnson transformation. Then, we confirmed that the residuals obtained from the result of ANOVA followed the normal distribution by Lilliefors test for normality. We also conducted pairwise comparisons (Duncan) to establish the statistically significant differences between each level of age group and hair oiliness.

\section{RESULTS}

\section{Participants}

A total of 432 participants (178 males and 254 females) completed the study with shampoo. And 370 (139 males and 231 females) completed with rinse (wash-out conditioner), while 161 (138 males and 23 females) used hair wax; 104 (63 males and 41 females) used hair gel; 307 (98 males and 209 females) used hair essence; 54 (22 males and 32 females) used hair tonic; and 207 (137 males and 70 females) tested with hair spray.

\section{Simple comparison of usage pattern by sex}

As summarized in Table 1 and Fig. 1, evaluation of the mean amount per daily use $(\mathrm{g} / \mathrm{d})$ of hair care products suggested that females consumed significantly larger amounts 
of shampoo and rinse than males $(p<0.05)$. For females, there is a positive correlation between hair length and daily shampoo and rinse usage (Fig. 2). Male groups used more hair gel and spray than female groups $(p<0.05)$ (Fig. 1). There was no sex difference in other hair care products (wax, spray, tonic, and essence). Especially, in the analysis of daily usage according to hair length $(\mathrm{g} / \mathrm{cm} / \mathrm{d})$, males used all of the seven hair care products more frequently than females $(p<0.05)$ (Table 1$)$.

\section{Usage pattern of hair care product by aging}

\section{1) Males}

There was no difference in the total amount of daily use of seven hair care products among the age groups. Significant correlation was observed between age and essence usage per unit length (ANOVA, $p<0.05$ ) (Table 2). After the data transformation, meaningful correlation was also observed in wax and spray usage per unit length (ANOVA, $p<0.05)$. It was found that the usage of over 30-year-old males was significantly higher than that of below 20 s.

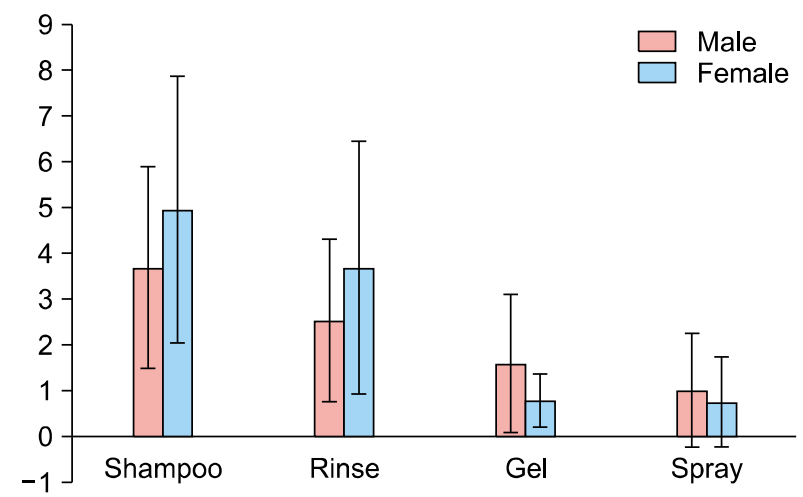

Fig. 1. Comparison of daily usage of hair care products $(\mathrm{g} / \mathrm{d})$ by sex.

Table 1. Daily use of hair care products and daily use per unit length of hair care products in Korean

\begin{tabular}{|c|c|c|c|c|c|c|c|c|}
\hline Product & Sex & Participants & Mean \pm SD (g) & $\begin{array}{l}\text { Estimated } \\
\text { mean (g) }\end{array}$ & $\begin{array}{l}\text { Geometric } \\
\text { mean (g) }\end{array}$ & P50 & P90 & P95 \\
\hline \multicolumn{9}{|l|}{ Daily use } \\
\hline \multirow{2}{*}{ Shampoo $(n=432)$} & Male & 178 & $3.677 \pm 2.201$ & 3.224 & 3.136 & 3.110 & 6.367 & 8.110 \\
\hline & Female & 254 & $4.938 \pm 2.904 *$ & 4.438 & 4.229 & 4.418 & 8.119 & 10.461 \\
\hline \multirow[t]{2}{*}{ Rinse $(n=370)$} & Male & 139 & $2.547 \pm 1.769$ & 2.085 & 1.913 & 2.260 & 4.493 & 5.848 \\
\hline & Female & 231 & $3.679 \pm 2.742 *$ & 3.278 & 2.830 & 3.136 & 6.934 & 8.527 \\
\hline \multirow[t]{2}{*}{ Wax $(n=161)$} & Male & 138 & $0.391 \pm 0.438$ & 0.275 & 0.251 & 0.269 & 0.772 & 1.126 \\
\hline & Female & 23 & $0.566 \pm 0.705$ & 0.251 & 0.264 & 0.289 & 1.181 & 2.100 \\
\hline \multirow[t]{2}{*}{ Gel $(n=104)$} & Male & 63 & $1.598 \pm 1.501 *$ & 1.238 & 1.086 & 1.148 & 3.075 & 3.307 \\
\hline & Female & 41 & $0.783 \pm 0.579$ & 0.657 & 0.547 & 0.657 & 1.605 & 1.911 \\
\hline \multirow[t]{2}{*}{ Essence $(\mathrm{n}=307)$} & Male & 98 & $0.597 \pm 0.648$ & 0.456 & 0.385 & 0.415 & 1.228 & 1.357 \\
\hline & Female & 209 & $0.657 \pm 0.560$ & 0.505 & 0.470 & 0.486 & 1.432 & 1.744 \\
\hline \multirow[t]{2}{*}{ Tonic $(n=54)$} & Male & 22 & $0.921 \pm 0.902$ & 0.714 & 0.623 & 0.559 & 1.464 & 2.670 \\
\hline & Female & 32 & $0.696 \pm 0.898$ & 0.507 & 0.401 & 0.418 & 1.300 & 1.508 \\
\hline \multirow[t]{2}{*}{ Spray $(n=207)$} & Male & 137 & $1.008 \pm 1.247^{*}$ & 0.597 & 0.524 & 0.564 & 2.328 & 4.153 \\
\hline & Female & 70 & $0.749 \pm 0.984$ & 0.380 & 0.351 & 0.443 & 1.856 & 2.823 \\
\hline \multicolumn{9}{|c|}{ Daily use per unit length } \\
\hline \multirow[t]{2}{*}{ Shampoo $(n=432)$} & Male & 178 & $0.453 \pm 0.316^{*}$ & 0.351 & 0.364 & 0.407 & 0.799 & 1.029 \\
\hline & Female & 254 & $0.155 \pm 0.123$ & 0.139 & 0.125 & 0.126 & 0.268 & 0.408 \\
\hline \multirow[t]{2}{*}{ Rinse $(n=370)$} & Male & 139 & $0.315 \pm 0.243 *$ & 0.215 & 0.221 & 0.260 & 0.660 & 0.737 \\
\hline & Female & 231 & $0.113 \pm 0.106$ & 0.101 & 0.082 & 0.088 & 0.201 & 0.272 \\
\hline \multirow[t]{2}{*}{ Wax $(n=161)$} & Male & 138 & $0.049 \pm 0.070^{*}$ & 0.033 & 0.028 & 0.030 & 0.107 & 0.125 \\
\hline & Female & 23 & $0.016 \pm 0.017$ & 0.007 & 0.008 & 0.008 & 0.040 & 0.051 \\
\hline \multirow[t]{2}{*}{ Gel $(n=104)$} & Male & 63 & $0.218 \pm 0.270^{*}$ & 0.149 & 0.128 & 0.136 & 0.403 & 0.609 \\
\hline & Female & 41 & $0.025 \pm 0.028$ & 0.017 & 0.016 & 0.018 & 0.037 & 0.059 \\
\hline \multirow[t]{2}{*}{ Essence $(n=307)$} & Male & 98 & $0.068 \pm 0.079 *$ & 0.047 & 0.040 & 0.039 & 0.158 & 0.197 \\
\hline & Female & 209 & $0.018 \pm 0.017$ & 0.014 & 0.012 & 0.012 & 0.040 & 0.048 \\
\hline \multirow[t]{2}{*}{ Tonic $(n=54)$} & Male & 22 & $0.096 \pm 0.080^{*}$ & 0.096 & 0.068 & 0.058 & 0.189 & 0.260 \\
\hline & Female & 32 & $0.023 \pm 0.027$ & 0.016 & 0.012 & 0.011 & 0.047 & 0.072 \\
\hline \multirow[t]{2}{*}{ Spray $(n=207)$} & Male & 137 & $0.116 \pm 0.148^{*}$ & 0.065 & 0.058 & 0.053 & 0.300 & 0.414 \\
\hline & Female & 70 & $0.022 \pm 0.038$ & 0.011 & 0.009 & 0.012 & 0.039 & 0.063 \\
\hline
\end{tabular}

SD: standard deviation. *Statistical significance in 3-way ANOVA, $p<0.05$. 
Daily amount of shampoo in females

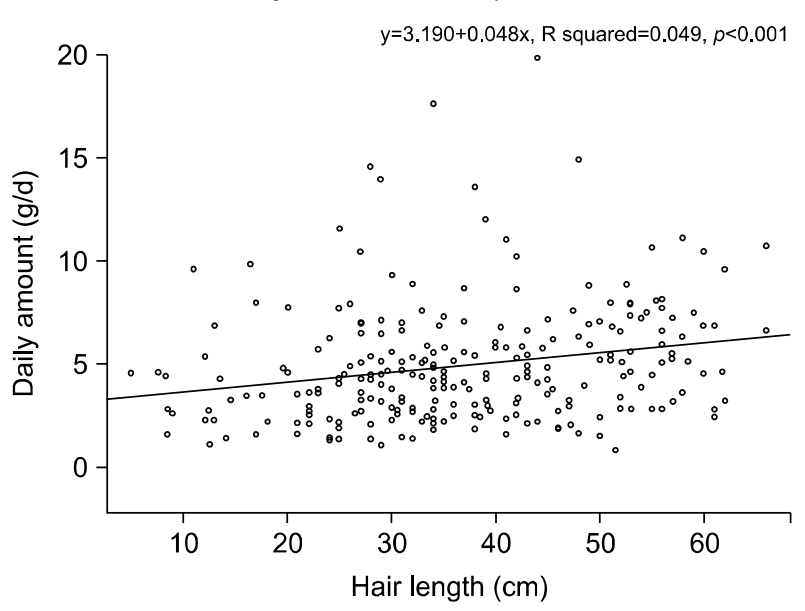

Daily amount of rinse in females

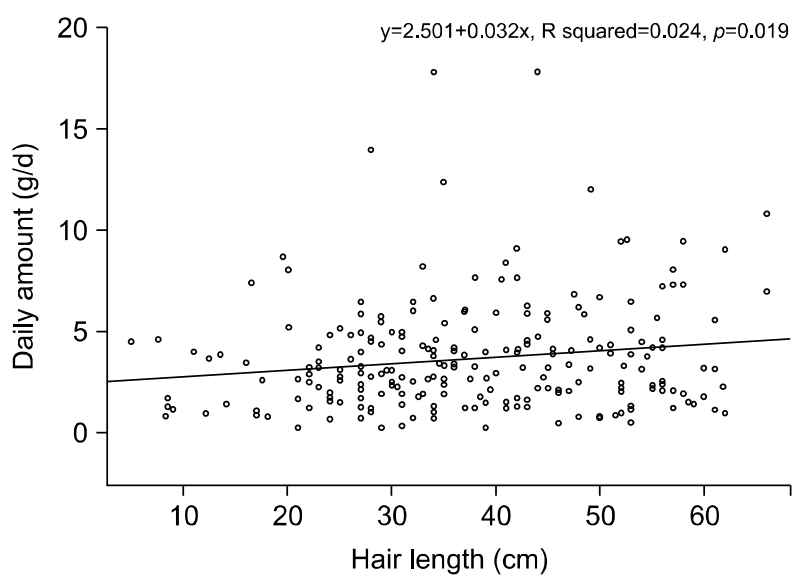

Daily amount of shampoo in males

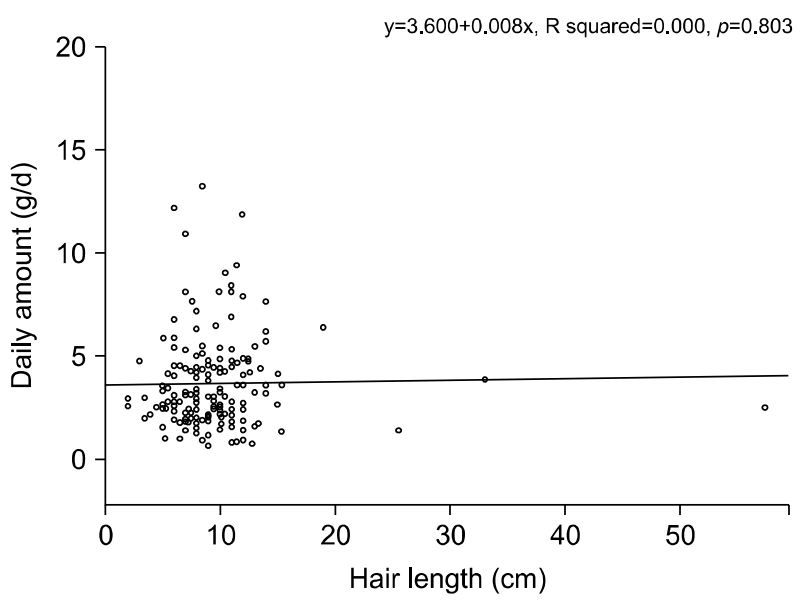

Daily amount of rinse in males

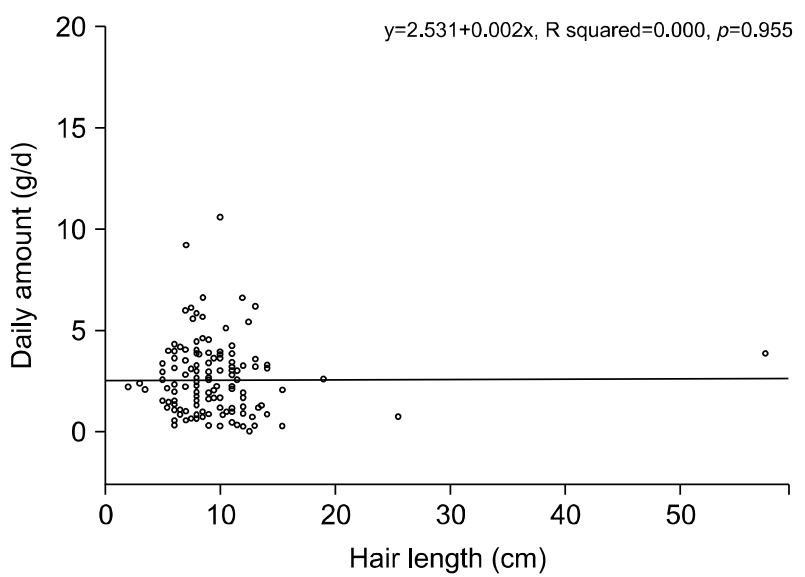

Fig. 2. Daily shampoo and rinse usage of male and female according to hair length.

These results were not confirmed in the fifties of wax user because of the small number of subjects.

\section{2) Females}

In daily shampoo usage $(\mathrm{g} / \mathrm{d})$, there was a significant difference among the age groups but no difference in other products (ANOVA, $p<0.001$ ). Daily usage of shampoo decreased by age (Fig. 3). Daily usage of hair essence per hair length $(\mathrm{g} / \mathrm{cm} / \mathrm{d})$ tended to increase with aging $(p<$ 0.001 ) but no correlation was found with the other hair products (Table 2).

\section{Usage pattern according to hair oiliness}

\section{1) Males}

There was statistical difference in the usage per day $(\mathrm{g} / \mathrm{d})$ or per unit length $(\mathrm{g} / \mathrm{cm} / \mathrm{d})$ of hair rinse according to the degree of hair oiliness (Table 3). Males with oily hair used more rinse than males with dry hair $(p<0.05)$.

\section{2) Females}

We found that females with oily hair type used more shampoo daily than other hair types $(p<0.05)$. However, the usage per day $(\mathrm{g} / \mathrm{d})$ or per unit length hair $(\mathrm{g} / \mathrm{cm} / \mathrm{d})$ of the hair conditioners (rinse, tonic, and essence) or hair styling products (wax, gel, and spray) did not differ according to the hair oiliness (Table 3).

\section{DISCUSSION}

Reliable exposure information for personal care products is needed to conduct safety assessments ${ }^{2}$. It is applied to risk assessment of cosmetic ingredients and safety evaluation of the products, including risk assessment on the unavoidable materials which were introduced by the manufacturing process of ingredients and products. Personal care products are generally applied to human skin and mainly produce local exposure, although use in the mucosal surfaces, lips, and eyes may also produce human 
Table 2. Daily use or daily use per unit length of hair care products according to age groups (male and female)

\begin{tabular}{|c|c|c|c|c|c|c|c|c|}
\hline Amount & Age $(y r)$ & Shampoo & Rinse & Wax & Gel & Essence & Tonic & Spray \\
\hline \multicolumn{9}{|l|}{ Male } \\
\hline \multirow[t]{5}{*}{ Daily use $(\mathrm{g} / \mathrm{d})$} & $10 \sim 19$ & - & - & $\begin{array}{c}0.33 \pm 0.39 \\
(n=14)\end{array}$ & $\begin{array}{c}0.94 \pm 0.49 \\
(n=7)\end{array}$ & $\begin{array}{l}0.46 \pm 0.36 \\
\quad(n=15)\end{array}$ & $\begin{array}{r}2.73 \\
(\mathrm{n}=1)\end{array}$ & $\begin{array}{c}0.64 \pm 0.99 \\
(n=14)\end{array}$ \\
\hline & $20 \sim 29$ & $\begin{array}{c}4.18 \pm 1.82 \\
\quad(n=45)\end{array}$ & $\begin{array}{l}2.34 \pm 2.04 \\
(\mathrm{n}=35)\end{array}$ & $\begin{array}{c}0.34 \pm 0.50 \\
(n=53)\end{array}$ & $\begin{array}{c}1.51 \pm 1.03 \\
(\mathrm{n}=11)\end{array}$ & $\begin{array}{c}0.56 \pm 0.61 \\
(\mathrm{n}=35)\end{array}$ & $\begin{array}{c}0.92 \pm 0.43 \\
(\mathrm{n}=4)\end{array}$ & $\begin{array}{c}1.03 \pm 1.43 \\
(\mathrm{n}=51)\end{array}$ \\
\hline & $30 \sim 39$ & $\begin{array}{l}3.78 \pm 2.69 \\
\quad(n=44)\end{array}$ & $\begin{array}{l}2.72 \pm 1.84 \\
\quad(n=32)\end{array}$ & $\begin{array}{c}0.47 \pm 0.44 \\
(\mathrm{n}=50)\end{array}$ & $\begin{array}{l}1.65 \pm 1.47 \\
(\mathrm{n}=15)\end{array}$ & $\begin{array}{l}0.52 \pm 0.46 \\
\quad(n=28)\end{array}$ & $\begin{array}{c}0.97 \pm 1.37 \\
(\mathrm{n}=7)\end{array}$ & $\begin{array}{c}1.03 \pm 1.20 \\
(n=43)\end{array}$ \\
\hline & $40 \sim 49$ & $\begin{array}{c}3.19 \pm 1.93 \\
(n=49)\end{array}$ & $\begin{array}{l}2.51 \pm 1.25 \\
\quad(\mathrm{n}=38)\end{array}$ & $\begin{array}{c}0.39 \pm 0.27 \\
(\mathrm{n}=19)\end{array}$ & $\begin{array}{c}1.86 \pm 2.11 \\
(n=19)\end{array}$ & $\begin{array}{c}0.88 \pm 1.11 \\
(n=16)\end{array}$ & $\begin{array}{c}0.47 \pm 0.22 \\
(\mathrm{n}=5)\end{array}$ & $\begin{array}{c}0.96 \pm 1.02 \\
(\mathrm{n}=22)\end{array}$ \\
\hline & $\geq 50$ & $\begin{array}{c}3.58 \pm 2.25 \\
\quad(n=40)\end{array}$ & $\begin{array}{c}2.64 \pm 1.95 \\
(\mathrm{n}=34)\end{array}$ & $\begin{array}{c}0.17 \pm 0.14 \\
(\mathrm{n}=2)\end{array}$ & $\begin{array}{c}1.59 \pm 1.13 \\
(n=11)\end{array}$ & $\begin{array}{c}0.80 \pm 0.31 \\
(n=4)\end{array}$ & $\begin{array}{c}0.94 \pm 0.43 \\
(n=5)\end{array}$ & $\begin{array}{c}1.58 \pm 1.32 \\
(\mathrm{n}=7)\end{array}$ \\
\hline \multirow[t]{5}{*}{$\begin{array}{l}\text { Daily use per unit } \\
\text { length }(\mathrm{g} / \mathrm{cm} / \mathrm{d})\end{array}$} & $10 \sim 19$ & - & - & $\begin{array}{c}0.03 \pm 0.04 * \\
(n=14)\end{array}$ & $\begin{array}{c}0.08 \pm 0.05 \\
(\mathrm{n}=7)\end{array}$ & $\begin{array}{c}0.04 \pm 0.03 * \\
(n=15)\end{array}$ & $\begin{array}{c}0.30 \\
(\mathrm{n}=1)\end{array}$ & $\begin{array}{c}0.07 \pm 0.12 * \\
\quad(n=14)\end{array}$ \\
\hline & $20 \sim 29$ & $\begin{array}{c}0.45 \pm 0.25 \\
\quad(n=45)\end{array}$ & $\begin{array}{l}0.26 \pm 0.24 \\
(\mathrm{n}=35)\end{array}$ & $\begin{array}{l}0.04 \pm 0.08^{*} \\
(\mathrm{n}=53)\end{array}$ & $\begin{array}{c}0.16 \pm 0.12 \\
(n=11)\end{array}$ & $\begin{array}{c}0.06 \pm 0.07^{*} \\
(\mathrm{n}=35)\end{array}$ & $\begin{array}{c}0.11 \pm 0.06 \\
(n=4)\end{array}$ & $\begin{array}{c}0.10 \pm 0.15^{*} \\
(\mathrm{n}=51)\end{array}$ \\
\hline & $30 \sim 39$ & $\begin{array}{c}0.43 \pm 0.29 \\
(\mathrm{n}=44)\end{array}$ & $\begin{array}{c}0.31 \pm 0.23 \\
(\mathrm{n}=32)\end{array}$ & $\begin{array}{l}0.06 \pm 0.07^{*} \\
\quad(\mathrm{n}=50)\end{array}$ & $\begin{array}{l}0.22 \pm 0.18 \\
\quad(n=15)\end{array}$ & $\begin{array}{l}0.06 \pm 0.05^{*} \\
(\mathrm{n}=28)\end{array}$ & $\begin{array}{c}0.08 \pm 0.09 \\
(\mathrm{n}=7)\end{array}$ & $\begin{array}{l}0.13 \pm 0.16^{*} \\
(\mathrm{n}=43)\end{array}$ \\
\hline & $40 \sim 49$ & $\begin{array}{c}0.45 \pm 0.33 \\
(\mathrm{n}=49)\end{array}$ & $\begin{array}{c}0.36 \pm 0.24 \\
(\mathrm{n}=38)\end{array}$ & $\begin{array}{c}0.06 \pm 0.04 * \\
(n=19)\end{array}$ & $\begin{array}{l}0.26 \pm 0.31 \\
\quad(n=19)\end{array}$ & $\begin{array}{l}0.12 \pm 0.11 * \\
\quad(n=16)\end{array}$ & $\begin{array}{c}0.06 \pm 0.02 \\
(\mathrm{n}=5)\end{array}$ & $\begin{array}{c}0.14 \pm 0.13^{*} \\
\quad(\mathrm{n}=22)\end{array}$ \\
\hline & $\geq 50$ & $\begin{array}{c}0.49 \pm 0.38 \\
(n=40)\end{array}$ & $\begin{array}{c}0.34 \pm 0.27 \\
(n=34)\end{array}$ & $\begin{array}{c}0.04 \pm 0.03^{*} \\
(\mathrm{n}=2)\end{array}$ & $\begin{array}{c}0.30 \pm 0.43 \\
(n=11)\end{array}$ & $\begin{array}{c}0.15 \pm 0.15^{*} \\
(n=4)\end{array}$ & $\begin{array}{c}0.11 \pm 0.06 \\
(n=5)\end{array}$ & $\begin{array}{c}0.16 \pm 0.13^{*} \\
(\mathrm{n}=7)\end{array}$ \\
\hline \multicolumn{9}{|l|}{ Female } \\
\hline \multirow[t]{5}{*}{ Daily use (g/d) } & $10 \sim 19$ & $\begin{array}{l}6.51 \pm 4.15^{*} \\
(\mathrm{n}=48)\end{array}$ & $\begin{array}{l}4.41 \pm 4.27 \\
\quad(n=44)\end{array}$ & - & $\begin{array}{c}0.85 \pm 0.25 \\
(\mathrm{n}=3)\end{array}$ & $\begin{array}{c}0.81 \pm 0.74 \\
(\mathrm{n}=32)\end{array}$ & $\begin{array}{r}0.54 \\
(\mathrm{n}=1)\end{array}$ & $\begin{array}{c}0.51 \pm 0.76 \\
(n=5)\end{array}$ \\
\hline & $20 \sim 29$ & $\begin{array}{c}5.58 \pm 2.45^{*} \\
(\mathrm{n}=51)\end{array}$ & $\begin{array}{c}3.97 \pm 2.30 \\
(\mathrm{n}=50)\end{array}$ & $\begin{array}{c}0.53 \pm 0.61 \\
(\mathrm{n}=3)\end{array}$ & $\begin{array}{c}0.68 \pm 0.64 \\
(n=11)\end{array}$ & $\begin{array}{c}0.60 \pm 0.40 \\
(n=64)\end{array}$ & $\begin{array}{c}0.48 \pm 0.52 \\
(n=6)\end{array}$ & $\begin{array}{c}0.61 \pm 0.63 \\
(\mathrm{n}=20)\end{array}$ \\
\hline & $30 \sim 39$ & $\begin{array}{l}4.47 \pm 2.04 * \\
\quad(n=54)\end{array}$ & $\begin{array}{l}3.52 \pm 2.10 \\
\quad(\mathrm{n}=52)\end{array}$ & $\begin{array}{c}0.81 \pm 0.91 \\
(\mathrm{n}=11)\end{array}$ & $\begin{array}{c}0.79 \pm 0.60 \\
(n=13)\end{array}$ & $\begin{array}{c}0.60 \pm 0.57 \\
(n=58)\end{array}$ & $\begin{array}{c}0.82 \pm 1.50 \\
(n=10)\end{array}$ & $\begin{array}{c}1.03 \pm 1.42 \\
(\mathrm{n}=24)\end{array}$ \\
\hline & $40 \sim 49$ & $\begin{array}{l}4.44 \pm 2.20^{*} \\
\quad(\mathrm{n}=53)\end{array}$ & $\begin{array}{c}3.84 \pm 2.52 \\
(\mathrm{n}=46)\end{array}$ & $\begin{array}{c}0.22 \pm 0.22 \\
(\mathrm{n}=7)\end{array}$ & $\begin{array}{c}0.87 \pm 0.58 \\
(n=11)\end{array}$ & $\begin{array}{c}0.61 \pm 0.56 \\
(\mathrm{n}=41)\end{array}$ & $\begin{array}{c}0.62 \pm 0.42 \\
(\mathrm{n}=9)\end{array}$ & $\begin{array}{c}0.64 \pm 0.77 \\
(n=13)\end{array}$ \\
\hline & $\geq 50$ & $\begin{array}{c}3.76 \pm 2.90^{*} \\
(\mathrm{n}=48)\end{array}$ & $\begin{array}{l}2.51 \pm 1.56 \\
\quad(\mathrm{n}=39)\end{array}$ & $\begin{array}{c}0.49 \pm 0.29 \\
(\mathrm{n}=2)\end{array}$ & $\begin{array}{c}0.74 \pm 0.81 \\
(n=3)\end{array}$ & $\begin{array}{l}0.96 \pm 0.57 \\
(n=14)\end{array}$ & $\begin{array}{c}0.84 \pm 0.56 \\
(n=6)\end{array}$ & $\begin{array}{c}0.61 \pm 0.32 \\
(\mathrm{n}=8)\end{array}$ \\
\hline \multirow[t]{5}{*}{$\begin{array}{l}\text { Daily use per unit } \\
\text { length }(\mathrm{g} / \mathrm{cm} / \mathrm{d})\end{array}$} & $10 \sim 19$ & $\begin{array}{l}0.18 \pm 0.13 \\
\quad(n=48)\end{array}$ & $\begin{array}{c}0.12 \pm 0.13 \\
(\mathrm{n}=44)\end{array}$ & - & $\begin{array}{c}0.02 \pm 0.01 \\
(n=3)\end{array}$ & $\begin{array}{c}0.02 \pm 0.02 * \\
(\mathrm{n}=32)\end{array}$ & $\begin{array}{c}0.01 \\
(\mathrm{n}=1)\end{array}$ & $\begin{array}{c}0.01 \pm 0.01 \\
(n=5)\end{array}$ \\
\hline & $20 \sim 29$ & $\begin{array}{c}0.12 \pm 0.06 \\
(\mathrm{n}=51)\end{array}$ & $\begin{array}{c}0.09 \pm 0.05 \\
(n=50)\end{array}$ & $\begin{array}{c}0.01 \pm 0.01 \\
(n=3)\end{array}$ & $\begin{array}{c}0.01 \pm 0.01 \\
(n=11)\end{array}$ & $\begin{array}{l}0.01 \pm 0.01 * \\
\quad(n=64)\end{array}$ & $\begin{array}{c}0.01 \pm 0.02 \\
(n=6)\end{array}$ & $\begin{array}{c}0.01 \pm 0.01 \\
(\mathrm{n}=20)\end{array}$ \\
\hline & $30 \sim 39$ & $\begin{array}{c}0.12 \pm 0.07 \\
(n=54)\end{array}$ & $\begin{array}{c}0.09 \pm 0.06 \\
(\mathrm{n}=52)\end{array}$ & $\begin{array}{c}0.02 \pm 0.02 \\
(\mathrm{n}=11)\end{array}$ & $\begin{array}{c}0.02 \pm 0.01 \\
(n=13)\end{array}$ & $\begin{array}{l}0.02 \pm 0.02 * \\
\quad(n=58)\end{array}$ & $\begin{array}{c}0.02 \pm 0.04 \\
(n=10)\end{array}$ & $\begin{array}{c}0.03 \pm 0.06 \\
(\mathrm{n}=24)\end{array}$ \\
\hline & $40 \sim 49$ & $\begin{array}{l}0.16 \pm 0.10 \\
(\mathrm{n}=53)\end{array}$ & $\begin{array}{l}0.13 \pm 0.10 \\
(\mathrm{n}=46)\end{array}$ & $\begin{array}{c}0.01 \pm 0.02 \\
(\mathrm{n}=7)\end{array}$ & $\begin{array}{c}0.04 \pm 0.04 \\
(n=11)\end{array}$ & $\begin{array}{l}0.02 \pm 0.02 * \\
\quad(n=41)\end{array}$ & $\begin{array}{c}0.02 \pm 0.02 \\
(\mathrm{n}=9)\end{array}$ & $\begin{array}{c}0.02 \pm 0.02 \\
(n=13)\end{array}$ \\
\hline & $\geq 50$ & $\begin{array}{l}0.20 \pm 0.20 \\
\quad(n=48)\end{array}$ & $\begin{array}{c}0.14 \pm 0.17 \\
(\mathrm{n}=39)\end{array}$ & $\begin{array}{c}0.02 \pm 0.02 \\
(n=2)\end{array}$ & $\begin{array}{c}0.04 \pm 0.06 \\
(\mathrm{n}=3)\end{array}$ & $\begin{array}{c}0.04 \pm 0.03^{*} \\
(n=14)\end{array}$ & $\begin{array}{c}0.04 \pm 0.03 \\
(n=6)\end{array}$ & $\begin{array}{c}0.03 \pm 0.01 \\
(n=8)\end{array}$ \\
\hline
\end{tabular}

Values are presented as mean \pm standard deviation. -: not checked. ${ }^{*}$ Statistical significance in ANOVA, $p<0.05$.

systemic exposure. In the European Union, US, and Japan, the safety of personal care products is regulated under cosmetic and/or drug regulations ${ }^{13}$. The most important process of risk assessment is the exposure assessment. Exposure assessment is the process of calculating how much our body is exposed to external materials such as the use of cosmetics. It takes into consideration of factors such as the concentration of the ingredients in the product, the amount, frequency, and method of using, skin type of user, and so on. This is followed by the calculation of Systemic Exposure Dosage, which is important factor of calculating of Margin of Safety. However, information sources remain scattered ${ }^{14}$. A few previous reports ${ }^{2-7,9,10,12,15,16}$ restricted the exposure data of hair care products to shampoos and rinse-off conditioners. Recently, a paper has been published confirming the exposure of hair cosmetic products using a probabilistic assessment in the French population $^{8}$. In this study, exposure data for hair care pro- 
ducts were extended to 7 items ( 1 shampoo, 3 hair conditioners, and 3 hair styling products) and their daily usage was investigated. It is important to simulate or set up conditions that mimic the daily usage of the products for accurate evaluation of their usage pattern exactly. Previous reports consisted of face-to-face surveys or delivery and

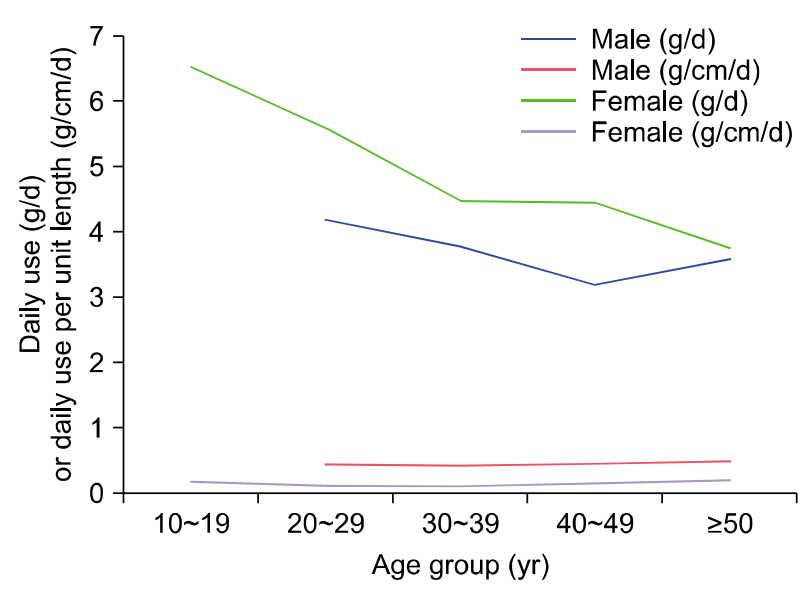

Fig. 3. Daily use $(g / d)$ or daily use per unit length $(g / c m / d)$ of hair shampoo according to age. collection methods of test products to determine the usage for a specific time, which were of limited value in estimating the actual use. We sought to determine the "real world" exposure data of hair care products, and therefore, we confirmed pre- and post-weighing of all the used products over a two-week period using a precision balance. Therefore, in this study, we investigated the practical usage. Notably, the studies varied according to the geographic regions, ethnic or cultural preferences, and the study design ${ }^{2,3,5-7,12}$. The present data represent the Korean usage pattern.

Throughout this study, the daily average amounts of shampoo and rinse increased in females, especially young females. However, this condition disappeared when calibrated according to unit length. In the study of Loretz et al. $^{2,3}$, the average amount of shampoo and conditioner used increased as the reported length of the females' hair increased. Females usually have longer hairs than males, and the hair length affected the daily usage of shampoo and rinse. Interestingly, all the hair care products studied scored higher levels of usage among males when calibrated per unit length. This reason might be due to the container and/or dispenser pump of the hair care products.

Table 3. Daily use or daily use per unit length of hair care products according to hair oiliness (male and female)

\begin{tabular}{|c|c|c|c|c|c|c|c|c|}
\hline Amount & Category & Shampoo & Rinse & Wax & Gel & Essence & Tonic & Spray \\
\hline \multicolumn{9}{|l|}{ Male } \\
\hline \multirow[t]{3}{*}{ Daily use (g/d) } & Dry & $\begin{array}{l}2.91 \pm 1.68 \\
(\mathrm{n}=29)\end{array}$ & $\begin{array}{c}1.89 \pm 1.10^{*} \\
(\mathrm{n}=21)\end{array}$ & $\begin{array}{c}0.51 \pm 0.43 \\
(\mathrm{n}=12)\end{array}$ & $\begin{array}{c}2.21 \pm 0.96 \\
(\mathrm{n}=8)\end{array}$ & $\begin{array}{l}0.81 \pm 1.07 \\
\quad(n=10)\end{array}$ & & $\begin{array}{c}1.29 \pm 1.46 \\
(n=14)\end{array}$ \\
\hline & Neutral & $\begin{array}{c}3.78 \pm 2.23 \\
\quad(\mathrm{n}=83)\end{array}$ & $\begin{array}{c}2.42 \pm 1.78^{*} \\
(n=64)\end{array}$ & $\begin{array}{c}0.39 \pm 0.31 \\
(\mathrm{n}=52)\end{array}$ & $\begin{array}{c}1.88 \pm 2.14 \\
(\mathrm{n}=22)\end{array}$ & $\begin{array}{c}0.62 \pm 0.50 \\
(\mathrm{n}=35)\end{array}$ & $\begin{array}{l}1.21 \pm 1.30 \\
(\mathrm{n}=9)\end{array}$ & $\begin{array}{c}1.03 \pm 1.29 \\
(\mathrm{n}=49)\end{array}$ \\
\hline & Oily & $\begin{array}{c}3.88 \pm 2.32 \\
(\mathrm{n}=66)\end{array}$ & $\begin{array}{c}2.95 \pm 1.89^{*} \\
(n=54)\end{array}$ & $\begin{array}{c}0.37 \pm 0.51 \\
(\mathrm{n}=74)\end{array}$ & $\begin{array}{c}1.26 \pm 0.95 \\
(\mathrm{n}=33)\end{array}$ & $\begin{array}{l}0.54 \pm 0.64 \\
(\mathrm{n}=53)\end{array}$ & $\begin{array}{c}0.72 \pm 0.44 \\
\quad(n=13)\end{array}$ & $\begin{array}{c}0.94 \pm 1.19 \\
(n=74)\end{array}$ \\
\hline \multirow[t]{3}{*}{$\begin{array}{l}\text { Daily use per unit } \\
\text { length }(\mathrm{g} / \mathrm{cm} / \mathrm{d})\end{array}$} & Dry & $\begin{array}{c}0.37 \pm 0.25 \\
(\mathrm{n}=29)\end{array}$ & $\begin{array}{c}0.23 \pm 0.14^{*} \\
(\mathrm{n}=21)\end{array}$ & $\begin{array}{c}0.06 \pm 0.06 \\
(n=12)\end{array}$ & $\begin{array}{c}0.27 \pm 0.14 \\
(\mathrm{n}=8)\end{array}$ & $\begin{array}{c}0.11 \pm 0.16 \\
(n=10)\end{array}$ & & $\begin{array}{c}0.17 \pm 0.20 \\
(n=14)\end{array}$ \\
\hline & Neutral & $\begin{array}{c}0.48 \pm 0.36 \\
(\mathrm{n}=83)\end{array}$ & $\begin{array}{c}0.31 \pm 0.25^{*} \\
(n=64)\end{array}$ & $\begin{array}{c}0.04 \pm 0.03 \\
(\mathrm{n}=52)\end{array}$ & $\begin{array}{l}0.25 \pm 0.35 \\
\quad(\mathrm{n}=22)\end{array}$ & $\begin{array}{l}0.07 \pm 0.07 \\
(\mathrm{n}=35)\end{array}$ & $\begin{array}{c}0.11 \pm 0.10 \\
(n=9)\end{array}$ & $\begin{array}{c}0.11 \pm 0.13 \\
(\mathrm{n}=49)\end{array}$ \\
\hline & Oily & $\begin{array}{c}0.46 \pm 0.28 \\
(n=66)\end{array}$ & $\begin{array}{c}0.35 \pm 0.26^{*} \\
(\mathrm{n}=54)\end{array}$ & $\begin{array}{c}0.05 \pm 0.09 \\
(n=74)\end{array}$ & $\begin{array}{l}0.18 \pm 0.23 \\
\quad(n=33)\end{array}$ & $\begin{array}{c}0.06 \pm 0.06 \\
(\mathrm{n}=53)\end{array}$ & $\begin{array}{c}0.09 \pm 0.06 \\
(n=13)\end{array}$ & $\begin{array}{c}0.11 \pm 0.15 \\
(\mathrm{n}=74)\end{array}$ \\
\hline \multicolumn{9}{|l|}{ Female } \\
\hline \multirow[t]{3}{*}{ Daily use (g/d) } & Dry & $\begin{array}{l}4.27 \pm 2.27 \\
\quad(\mathrm{n}=90)\end{array}$ & $\begin{array}{c}3.29 \pm 2.26 \\
(\mathrm{n}=77)\end{array}$ & $\begin{array}{c}0.39 \pm 0.44 \\
(n=4)\end{array}$ & $\begin{array}{c}0.69 \pm 0.47 \\
(\mathrm{n}=8)\end{array}$ & $\begin{array}{l}0.72 \pm 0.64 \\
(\mathrm{n}=29)\end{array}$ & $\begin{array}{c}0.58 \pm 0.32 \\
(n=5)\end{array}$ & $\begin{array}{c}0.95 \pm 1.43 \\
(n=14)\end{array}$ \\
\hline & Neutral & $\begin{array}{l}5.10 \pm 2.88 \\
(n=141)\end{array}$ & $\begin{array}{c}3.68 \pm 2.56 \\
(n=132)\end{array}$ & $\begin{array}{c}0.64 \pm 0.64 \\
(n=12)\end{array}$ & $\begin{array}{l}0.68 \pm 0.44 \\
\quad(n=15)\end{array}$ & $\begin{array}{c}0.67 \pm 0.55 \\
(\mathrm{n}=106)\end{array}$ & $\begin{array}{c}0.78 \pm 1.09 \\
(n=20)\end{array}$ & $\begin{array}{c}0.60 \pm 0.67 \\
(n=29)\end{array}$ \\
\hline & Oily & $\begin{array}{c}6.59 \pm 4.30^{*} \\
(\mathrm{n}=23)\end{array}$ & $\begin{array}{c}5.04 \pm 4.54 \\
(\mathrm{n}=22)\end{array}$ & $\begin{array}{c}0.54 \pm 0.98 \\
(n=7)\end{array}$ & $\begin{array}{l}0.91 \pm 0.71 \\
\quad(n=18)\end{array}$ & $\begin{array}{c}0.62 \pm 0.55 \\
\quad(n=74)\end{array}$ & $\begin{array}{c}0.53 \pm 0.49 \\
(\mathrm{n}=7)\end{array}$ & $\begin{array}{c}0.81 \pm 1.00 \\
(n=27)\end{array}$ \\
\hline \multirow[t]{3}{*}{$\begin{array}{l}\text { Daily use per unit } \\
\text { length }(\mathrm{g} / \mathrm{cm} / \mathrm{d})\end{array}$} & Dry & $\begin{array}{l}0.16 \pm 0.15 \\
\quad(\mathrm{n}=90)\end{array}$ & $\begin{array}{c}0.13 \pm 0.14 \\
(\mathrm{n}=77)\end{array}$ & $\begin{array}{c}0.01 \pm 0.01 \\
(n=4)\end{array}$ & $\begin{array}{c}0.03 \pm 0.03 \\
(\mathrm{n}=8)\end{array}$ & $\begin{array}{c}0.02 \pm 0.03 \\
(\mathrm{n}=29)\end{array}$ & $\begin{array}{c}0.03 \pm 0.02 \\
(n=5)\end{array}$ & $\begin{array}{c}0.03 \pm 0.03 \\
(n=14)\end{array}$ \\
\hline & Neutral & $\begin{array}{c}0.15 \pm 0.11 \\
(n=141)\end{array}$ & $\begin{array}{c}0.10 \pm 0.08 \\
(\mathrm{n}=132)\end{array}$ & $\begin{array}{c}0.02 \pm 0.01 \\
(\mathrm{n}=12)\end{array}$ & $\begin{array}{c}0.02 \pm 0.01 \\
(\mathrm{n}=15)\end{array}$ & $\begin{array}{c}0.02 \pm 0.02 \\
(n=106)\end{array}$ & $\begin{array}{c}0.02 \pm 0.03 \\
(\mathrm{n}=20)\end{array}$ & $\begin{array}{c}0.02 \pm 0.02 \\
(n=29)\end{array}$ \\
\hline & Oily & $\begin{array}{c}0.18 \pm 0.11 * \\
(\mathrm{n}=23)\end{array}$ & $\begin{array}{c}0.14 \pm 0.12 \\
(n=22)\end{array}$ & $\begin{array}{c}0.02 \pm 0.03 \\
(n=7)\end{array}$ & $\begin{array}{c}0.03 \pm 0.04 \\
(n=18)\end{array}$ & $\begin{array}{c}0.02 \pm 0.01 \\
(n=74)\end{array}$ & $\begin{array}{c}0.01 \pm 0.01 \\
(n=7)\end{array}$ & $\begin{array}{c}0.03 \pm 0.05 \\
(n=27)\end{array}$ \\
\hline
\end{tabular}

Values are presented as mean \pm standard deviation. ${ }^{*}$ Statistical significance in ANOVA, $p<0.05$. 
This point has been described by Gomez-Berrada et al. ${ }^{17}$. The authors showed that the consumption of cosmetic product was slightly higher when the product was packaged in tube with a flip top cap than in bottle with a pump $^{17}$. Most males in Korea have shorter hair than females. And, this fact could be rechecked in this study. The amount dispensed from container with just one push, is constant. Therefore, a single pumping action indicated a relative large amount for males or physiologically, and males pressed the pump more powerfully or frequently. The males's usage of hair care products was not different according to age group, but the usage of shampoo and rinse increased proportionately in younger females. In this study, we confirmed that Korean females had shorter hair as they grew older, which might be related to females's usage.

In daily usage per unit length, the use of essence was the highest in both males and females over 40 years of age. males's daily usage per unit length of wax and spray tended to increase with aging. Physiologically, hair volume tends to decrease after reaching a peak in the 40 's, suggesting that older people might use hair essence more than younger people to maintain their hair elasticity or volume $^{18}$.

The usage pattern of hair styling or conditioner products had no correlation with hair oiliness except rinse. Personal preferences or beauty might affect the choice of the styling products, and the health condition of hair shaft might dictate the choice of the conditioners used. Loretz et al. reported that females with oily, and/or curly hair used more shampoo ${ }^{2}$ and females with thick and/or curly hair used more conditioner ${ }^{3}$. We found that females with oily hair used more shampoo in the present study. In addition, additional ANOVA analysis showed that males with oily hair used the shampoo frequently. In our opinion, it is common sense that people with oily hair have a tendency to use more shampoo or conditioner. Compared with the Western data, Koreans tend to use lesser amount of rinse although their hairs are usually thicker than the Western hairs.

In conclusion, the present study provides basic information to derive real exposure data related to commonly used hair care products. The findings may enable risk assessment and development of safety guidelines for use of hair care products.

\section{ACKNOWLEDGMENT}

This study was supported by a grant (14172MFDS975) from the Ministry of Food and Drug Safety of Korea in 2016.

\section{CONFLICTS OF INTEREST}

The authors have nothing to disclose.

\section{ORCID}

Kyung-Duck Park, https://orcid.org/0000-0002-6067-7262

Byung Cheol Park, https://orcid.org/0000-0002-5449-8313

Ki Hyun Sung, https://orcid.org/0000-0002-8425-837X

Eunyoung Lee, https://orcid.org/0000-0001-5098-777X

Taewon Lee, https://orcid.org/0000-0003-4741-5054

Daeki Yoon, https://orcid.org/0000-0001-7668-1760

Kyu Bong Kim, https://orcid.org/0000-0003-2139-2296

Gyeong Hun Park, https://orcid.org/0000-0001-8890-8678

II Yong Park, https://orcid.org/0000-0003-1613-4209

Myung Hwa Kim, https://orcid.org/0000-0002-9072-201X

\section{REFERENCES}

1. Van Engelen JG, Heinemeyer G, Rodriguez C. Consumer exposure scenarios: development, challenges and possible solutions. J Expo Sci Environ Epidemiol 2007;17 Suppl 1: S26-S33.

2. Loretz L, Api AM, Barraj L, Burdick J, Davis de A, Dressler $W$, et al. Exposure data for personal care products: hairspray, spray perfume, liquid foundation, shampoo, body wash, and solid antiperspirant. Food Chem Toxicol 2006; 44:2008-2018.

3. Loretz LJ, Api AM, Babcock L, Barraj LM, Burdick J, Cater $K C$, et al. Exposure data for cosmetic products: facial cleanser, hair conditioner, and eye shadow. Food Chem Toxicol 2008;46:1516-1524.

4. Wu XM, Bennett DH, Ritz B, Cassady DL, Lee K, HertzPicciotto I. Usage pattern of personal care products in California households. Food Chem Toxicol 2010;48:31093119.

5. Biesterbos JW, Dudzina T, Delmaar CJ, Bakker MI, Russel FG, von Goetz N, et al. Usage patterns of personal care products: important factors for exposure assessment. Food Chem Toxicol 2013;55:8-17.

6. Hall B, Tozer S, Safford B, Coroama M, Steiling W, Leneveu-Duchemin $M C$, et al. European consumer exposure to cosmetic products, a framework for conducting population exposure assessments. Food Chem Toxicol 2007;45: 2097-2108

7. Hall B, Steiling W, Safford B, Coroama M, Tozer S, Firmani $C$, et al. European consumer exposure to cosmetic products, a framework for conducting population exposure assessments Part 2. Food Chem Toxicol 2011;49:408-422.

8. Ficheux AS, Bernard A, Chevillotte G, Dornic N, Roudot AC. Probabilistic assessment of exposure to hair cosmetic products by the French population. Food Chem Toxicol 2016;92:205-216.

9. Ficheux AS, Wesolek N, Chevillotte G, Roudot AC. Consumption of cosmetic products by the French population. 
First part: frequency data. Food Chem Toxicol 2015;78: 159-169.

10. Ficheux AS, Chevillotte G, Wesolek N, Morisset T, Dornic $N$, Bernard A, et al. Consumption of cosmetic products by the French population second part: amount data. Food Chem Toxicol 2016;90:130-141.

11. Dornic N, Ficheux AS, Roudot AC. Consumption of cosmetic products by the French population. Third part: product exposure amount. Food Chem Toxicol 2017;106: 209-222.

12. Park JY, Lee K, Hwang $\mathrm{Y}$, Kim JH. Determining the exposure factors of personal and home care products for exposure assessment. Food Chem Toxicol 2015;77:105-110.

13. Nohynek GJ, Antignac E, Re T, Toutain H. Safety assessment of personal care products/cosmetics and their ingredients. Toxicol Appl Pharmacol 2010;243:239-259.

14. Zaleski RT, Egeghy PP, Hakkinen PJ. Exploring global ex- posure factors resources for use in consumer exposure assessments. Int J Environ Res Public Health 2016;13:744.

15. Bennett DH, Wu XM, Teague $\mathrm{CH}$, Lee $\mathrm{K}$, Cassady DL, Ritz $B$, et al. Passive sampling methods to determine household and personal care product use. J Expo Sci Environ Epidemiol 2012;22:148-160.

16. Gomez-Berrada MP, Ficheux AS, Dahmoul Z, Roudot AC, Ferret PJ. Exposure assessment of family cosmetic products dedicated to babies, children and adults. Food Chem Toxicol 2017; 103:56-65.

17. Gomez-Berrada MP, Ficheux AS, Galonnier M, Rolfo JE, Rielland $A$, Guillou $S$, et al. Influence of the container on the consumption of cosmetic products. Food Chem Toxicol 2017; 109:230-236.

18. Mirmirani P. Hormonal changes in menopause: do they contribute to a 'midlife hair crisis' in women? Br J Dermatol 2011;165 Suppl 3:7-11. 